\title{
A Logic for Vagueness
}

\author{
John Slaney \\ Australian National University \\ John.Slaney@anu.edu.au
}

Received by Greg Restall

Published November 2, 2010

http://www.philosophy.unimelb.edu.au/ajl/2010

(C) 2010 John Slaney

\section{The Sorites PaRAdox}

In Dummett's important paper [I] on the sorites paradox it is suggested that the vagueness of observational predicates such as '... is red' or more obviously '. . looks red' generates an apparent incoherence: their use resembles a game governed by inconsistent rules. A similar incoherence is seen by Wright [I8, [7] as a real and serious threat to very ordinary ideas of how language works. Wright argues not that the use of vague predicates is incoherent but that it would be if the use of language were a practice in which the admissibility of moves were determined by rules whose general properties are discoverable by appeal to non-behavioural notions. But unless we do move from anecdotes about behaviour to just such rules, how are we to reason at all?

The incoherence in question is an outcome of the vagueness or tolerance of observational locutions, and would seem if established for them to spread to non-observational vague expressions like ' $\ldots$. is water' or '.. is a test tube' thus vitiating almost all of our attempts to use language consistently, even in science. On the face of it, vagueness is everywhere, whence such deep-seated incoherence would upset even such fragile understanding of semantics as we have gleaned from a century's work. The argument connecting vagueness to incoherence, therefore, strikes at the heart of logic: every philosophical logician is called upon to respond to it.

The sorites paradox, the "slippery slope argument" or the "paradox of the heap", is old and famous and wears an air of sophistry. One feels that the problem will vanish on exposure of the trivial trick involved. What is surprising is that it is so deep and difficult after all. An example or two will help focus the discussion.

\footnotetext{
${ }^{\mathrm{I}} \mathrm{How}$ long and thin must a piece of glassware be, or how polluted may a liquid be, before it no longer counts as a test tube or as water?
} 
EXAMPLE I Imagine a long coloured strip shading gradually from scarlet at one end to lemon-yellow at the other, divided into 250 thin segments 2 Let $R(x)$ mean that the $x$-th segment, numbering from the red end, looks red. Then clearly $R(1)$. Moreover, if $R(1)$ then $R(2)$, because there is no discriminable difference between them. Similarly, if $R(2)$ then $R(3) \ldots$ and so on. Hence, by a mere 249 applications of modus ponens, $\mathrm{R}(250)$.

EXAMPLE 2 Members of successive generations of animals belong to the same species, having very similar structure and genetic composition, being crossfertile, etc. Therefore chicken eggs can only be laid by chickens which can only come from chicken eggs. So neither came first: there have always been chickens, even in the precambrian before birds evolved.

EXAmple 3 Transition from one of the Ages of Man to another is a gradual matter. No-one becomes adolescent, elderly or middle-aged in one minute: these things creep up on one over several years. Since one is a child at the age of Iooo days, and since for any a if one is a child when a days old one is still a child at $a+1$ days old, one is always a child, even after 20,000 days.

The problem here is one of aggeregation: the natural view of the situation on the small scale does not cohere with that on the large scale. Predicates such as '. . looks red' or '... is a chicken' are tolerant of sufficiently small changes in the relevant respects (colour, genetics). That is, for example, it cannot be that of two colour patches no difference between which is directly discriminable 3 one looks red and the other does not. Large changes in the same respects, however, do make the difference between applicability and inapplicability of the predicates; yet the large changes are made up of the small ones.

Any solution to the paradox has to generate a response to a logical argument. In the first example above the argument consists of 250 premises and a conclusion though we might well extend it to get all the conditional premises from a generalisation

$$
\forall x(R(x) \rightarrow R(x+1))
$$

to the effect that no one small change is enough to make all the difference. There are only four possible solutions. We can:

(a) Deny that the problem is or can be legitimately set up. That is, hold that logic does not apply to vague expressions.

(b) Accept that logic does legitimately apply here but hold that this particular argument is invalid. That is, see this argument as a counter-example to modus ponens and so as a refutation of classical logic.

${ }^{2}$ There is no need to imagine: see http://users. cecs.anu.edu.au/ jks/sorites.html

${ }^{3}$ The difference between the colour patches is always in principle indirectly discriminable, for instance by noting that the one matches a third sample while the other does not. The sorites paradox is not really a problem about indiscernability, however, but about any differences too small to bear the weight of major distinctions. The "red" example could be re-worked with about 20 colours instead of 250 . 
(c) Accept both that logic applies in such cases and that the argument is valid but deny one of the premises.

(d) Accept the argument and its premises, and hence embrace the conclusion also.

None of these is especially attractive or easy to maintain. Option (a) is associated with Frege who famously held that a predicate with a fuzzy boundary of application would lack a sense and so could not be used in a sentence which achieved truth conditions. Unfortunately, natural languages like English or German are full of vagueness-yet evidently we do argue and theorise using natural language and we do apply criteria of rationality to each other's discourse in a reasonably stable way. To take option (a) is therefore to hold formal logic unfit for the purposes for which it was advertised. Such an attitude shows a lack of respect for the ordinary. Option (b) would require that we give up modus ponens, one of the intuitively best-attested argument forms. This too is in conflict with certain data regarding usage and with the fact that we find it hard to construe any connective as a conditional unless it allows that inference. Option (c) involves maintaining that no predicate is sorites-tolerant in the canvassed sense. On such an account a difference in colour too small to be perceptible could make all the difference between looking red and not looking red. This is extremely counter-intuitive. To say the least, it runs against such understanding as we have of how observational terms get their meanings and how they are actually used.

The version of option (c) most popular with philosophers is one which appeals to some apparatus of supervaluation. It is sometimes held that corresponding to every vague predicate is a more or less determinate range of acceptable sharpenings-interpretations as precise predicates with roughly the same extension. The truth involving a vague predicate is then just what comes out true on every acceptable sharpening. Formally what this proposal amounts to is the substitution of boolean-valued models for two-valued ones. ${ }^{4}$ Briefly, my response to this idea is that while relating a vague expression to the set of its acceptable sharpenings may well be good and useful the supervaluation technique is not. An instructive analogy is the theory of irrational numbers in real analysis: every irrational number can be approximated as close as you like by rationals, so we could adopt a supervaluational view that the truth about, say, $\sqrt{2}$ is what comes out true on all its acceptable rationalisations. This does not work because irrationality matters for truth in the object language. The sentence

$$
\exists x\left(x^{2}=2\right)
$$

${ }^{4}$ For a good presentation of a sophisticated account along these lines see $[5]$. For more recent views, a good starting point is $[\mathrm{I} 6$. 
is true of the reals but false on all rationalisations. Similarly in the case of vagueness

$$
\forall x(R(x) \rightarrow R(x+1))
$$

is true of redness but comes out plain false on all sharpenings. So the supervaluation account gets truth values wrong in some of the crucial cases.

It is worth pausing here to note another popular response to the effect that, whether or not logic can be applied to vague discourse, there is no need to apply it or to investigate its application to such discourse because in all cases that matter (e.g. science) vagueness is eliminable.5 Well in the first place it is not obvious that vagueness really is eliminable-maybe it is in physics, but most likely not in psychology for instance-and in the second place even if vague expressions can always be replaced by precise ones it is not clear that the "precisified" version of the language is in any way preferable. The prejudice in favour of precision is a legacy of outmoded philosophies of language and is long overdue for demolition. Finally, whatever the merits of restricting attention to precise languages this does not solve but merely ignores the problems arising with respect to sorites-susceptible languages like scientific English which after all fall within the intended scope of logical theory.

Dummett's paper hints at a prima facie argument for option (d). The sorites reasoning appears to supply a perfect case for applying a predicate like ' . . looks red' to any coloured object. This is obviously in conflict with other rules of our language which dictate the witholding of this predicate from lemons, grass and snow. For this reason, option (d) actually tends to collapse back into the discredited option (a), since on the face of it if the rules of our language are inconsistent then they are not (coherently) formalisable. Dummett says:

'Red' has to be a vague predicate if it is to be governed by the principle that, if I cannot discern any difference between the colour of $a$ and the colour of $b$, and I have characterised $a$ as red, then I am bound to accept a characterisation of $b$ as red. ... But by hypothesis one could force someone, faced with a sufficiently long series of objects, ... to admit that an object which was plainly blue ... was red. ... Hence it appears to follow that the use of any predicate which is taken as being governed by such a principle is potentially inconsistent: the inconsistency fails to come to light only because the principle is never sufficiently pressed. Thus Frege appears to be vindicated, and the use of vague predicates ... is intrinsically incoherent 6

The form of the argument is by elimination. Ruling out options (b) and (c) will leave us with no choice but (a) — except (d), which amounts to a version of (a). This paper is not concerned with arguments for or against option (c). What I

5 The locus classicus for this view is [9].

${ }^{6}[$ I], p. 264 of Truth and Other Enigmas. 
want to consider is Dummett's attempt to close the door on option (b). If I can wedge a foot in that door then the argument for the extraordinary conclusion that vaguness defeats logic will remain less than persuasive.

Obviously it will not do simply to reject the logical principle of detachment. An account is owed by anyone opting for a "deviant" logical solution of the sorites paradox of why, if modus ponens is invalid, everyone thinks it a paradigm of good reasoning. Dummett, in refusing to contemplate changing logic to deal with vagueness, throws out a sharper challenge. If we are to take route (b):

... we must declare the rule of universal instantiation invalid in the presence of vague predicates, or else regard modus ponens as invalid in that context. ... But either of these seems a desperate remedy, for the validity of these rules of inference seems absolutely constitutive of the meaning of 'every' and of 'if' 7

Well, I agree. An alternative logic has to give modus ponens a central place in determining the meaning of its implication connective, and of course it has to do this in such a way as to disallow the sorites argument. Yet the sorites works by modus ponens! Thus there is a very narrow line to tread. The purpose of the present paper is to indicate how it may be trodden.

\section{TOWARDS A DEVIANT LOGICAL SOLUTION}

There is a plausible argument to the effect that truth comes in degrees which I shall call the Simple Argument. No conclusions of this paper depend on it, but it does create a prima facie case for option (b). It runs as follows.

'Grass is green' is true (in English) if and only if grass is green. That is, the truth of 'Grass is green' marches together with the greenness of grass. But greenness is a matter of degree; so truth is a matter of degree.

As it stands, this is too simple to win many converts. Still, for the present let us take its conclusion seriously and examine some accounts of how logic might look were truth allowed to slide by degrees between the two poles of absolute truth and absolute falsehood.

A first shot is to adopt as the frame for truth-valuation the whole real unit interval instead of just its two end points, giving the continuum-valued logic of Łukasiewicz $]^{8}$ Like most first shots, this one misses, but before taking better

\footnotetext{
${ }^{7}$ Op cit, p. 252.

${ }^{8}$ Łukasiewicz himself does not seem to have proposed his continuum-valued logic as an account of vagueness, so the philosophical defects of such a proposal are not to be laid to his account.
} 
aim it is worth noting how it fares with respect to Dummett's challenge. The connectives are defined via functions on the unit interval:

$$
\begin{aligned}
a \wedge b & =\max (a, b) \\
a \vee b & =\min (a, b) \\
\sim a & =1-a \\
a \rightarrow b & =\max (b-a, 0)
\end{aligned}
$$

The numbers intuitively are "degrees of falsehood" or degrees by which propositions may fall short of absolute truth. The degree by which a conditional falls short of truth is just the increase in short-falling as we pass from its antecedent to its consequent. Propositions which are ordinarily assertible are those whose degree of falsehood is close to zero. This is itself a vague range-rightly so, for there is no exact boundary to the assertible-but the conditionals used to set up the sorites argument will fall well within it. There are then two versions of the sorites paradox, formulated with two versions of modus ponens obtained in turn from two ways of combining premises. Roughly, premises may be collected into a set and conjoined, or they may be collected into a multiset and added. There is a sort of intensional conjunction definable thus:

$$
A \oplus B=_{d f} \sim(A \rightarrow \sim B)
$$

The corresponding semantic clause is

$$
\mathrm{a} \oplus \mathrm{b}=\min (\mathrm{a}+\mathrm{b}, 1)
$$

Then the real modus ponens, constitutive of the meaning of 'if', is the argument form corresponding to the one-premise inference

$$
(\mathrm{A} \rightarrow \mathrm{B}) \oplus \mathrm{A} \text { therefore } \mathrm{B}
$$

This is perfectly valid, though easily confused with the superficially similar but invalid

$$
(A \rightarrow B) \wedge A \text { therefore } B
$$

If the sorites paradox is set up using real modus ponens, the present account solves it by insisting that although each individual conditional premise is assertible, being very close to true, in the combination of 249 of them the negligible short-fallings accumulate additively until the total falsehood is far from negligible. If the pseudo-modus ponens is used, the paradox is solved by holding the argument invalid as each of the 249 steps takes us further from the truth by a tiny amount. Dummett's challenge is at least addressed, in that modus ponens is not abandoned but merely refined. We shall see below that the challenge can be squarely met by a logic of this general sort, but first we should note in what other respects Łukasiewicz continuum-valued logic is unsatisfactory. 
The reasons why the Łukasiewicz story will not do are hardly new with this paper. Firstly, even if vagueness does require departure from the truth to come by degrees as the simple argument suggests, these are not much like numbers. It makes little sense to speak of one statement as being 46.92 times as far from the truth as another, and even less to speak of it as being 21.7\% false. That is to say, while the unit interval has the appeal of mathematically familiarity, it also has intrusive arithmetical properties which are meaningless for the intended interpretation but which affect the logic, for instance by validating inferences like that from $(A \rightarrow B) \rightarrow B$ to $(B \rightarrow A) \rightarrow A$. The total order of the unit interval is also unconvincing: why need we be able to say of the statement that Ioo grains of salt make a pinch and the statement that grass is brown that one is closer to the truth than the other? They would seem to differ in kind of departure from truth and not only in degree. Secondly, the simpleminded substitution of the unit interval for the truth values lays us open to the higher-order sorites paradoxes, for vague statements no more have precise degree-of-truth conditions than they have precise truth conditions. The sorites argument, then, can be re-worked with a second-order predicate like 'The degree of falsehood of the statement that $x$ is red is less than o.I' or 'It is falser to assert that $x$ is red than to deny it'.

Most fuzzy logicians attempt to deal with these objections by maintaining in some way that the assignments of values to statements are themselves fuzzy. So instead of assigning precise numbers from the real unit we are to assign more or less vaguely delimited ranges of such numbers, perhaps correlated with some natural language expressions like 'almost true' or 'fairly false'. This move, of making the metalogic fuzzy as well, has some success with the problem of higher-order paradoxes, but leaves unsolved that of intrusive arithmetical properties. Even at the syntactic level we may ask which forms of inference are the logically correct ones. Orthodox fuzzy logics either bite the bullet of continuing to hold all the principles validated by Łukasiewicz logic to be correct or bite the marshmallow of rejecting precise logical consequence altogether in favour of the idea that vagueness extends everywhere, giving us "approximately valid" reasoning at best. The former line I have suggested is over-strength and under-motivated. The latter is too much of a retreat. No difficulties raised by vagueness have been shown to affect straightforward reasoning steps like inferring conjunctions from their conjuncts or vice versa. Such healthy and blameless babies should not be thrown out simply in order to be rid of a spoonful of bathwater.

Still, fuzzy logic seems to have raised one valuable insight. There is a need for some concept of degree in the semantic theory, even if it should not be construed as numerical degree. And the diagnosis of the sorites problem is fundamentally right: trivially small removals from the absolute truth can accumulate as premises are applied to one another until the total aberration is far from trivial. The next pressing need, then, is to re-work the logic more 
carefully, preserving the insights and the ability to meet Dummett's challenge while avoiding the absurdities of the old fuzzy logic. The place to begin is in proof theory.

\section{The Logic F}

The place to begin in proof theory is with natural deduction. The system $\mathbf{F}$ (for "fuzzy") to be laid out here as a labelled deductive system is adapted from [13], to which reference should be made for more detailed explanation and motivation of the key ideas. As pointed out in [13], this way of formulating nonclassical logics is not new. To the list of precursors given there I would add Meyer's [7].

The objects with which the logic deals are labelled formulae (in fact, representing sequents in a fairly transparent way). These are ordered pairs each consisting of a structured identifier called its label and a single formula. An assumption is an object of the form

$$
\mathcal{L}_{\mathrm{A}}: A
$$

where $\mathcal{L}_{A}$ is the label identifying formula $A$. This corresponds to the sequent

$$
A \vdash A
$$

and may be introduced into any proof at any point. The rest of the rules, for introducing and eliminating logical constants, serve to transform such trivially valid sequents into nontrivially valid ones. As each assumption is made, the formula introduced will be given a short label: if we have occasion to write out proofs, we shall use numerals for this purpose, though the reader who feels that Greek letters or the like would look "more logical" is free to make substitutions to taste.

There are two differences between this account of system $\mathbf{F}$ and the corresponding labelled classical logic. The first is that negation in $\mathbf{F}$ is like that of intuitionist logic, so it lacks the classical rule of double negation elimination. The second is that, as in [13], we distinguish between two ways in which labels may be combined. Building on the insight won by the fuzzy logicians we allow ourselves to collect assumptions into sets just as classically, or into multisets. As presaged above, we should expect the premises of a sorites argument, and indeed those of modus ponens generally, to come in a multiset, while those of a purely extensional inference like adjunction would more naturally come in a set.

Because of the need to distinguish sets from multisets, we shall require two notations for the combination of labels. Each label represents a bunch of formulae, which may be either a set or a multiset of smaller bunches. For set union we use the comma ', and for multiset union the semicolon ';'. The two 
operations for combining labels may be nested inside each other to any finite depth, so we shall sometimes need to use parentheses in the normal way to disambiguate compounds. The notation

$$
\Gamma(\mathrm{X})
$$

stands for a label in which $X$ occurs as a sub-label. Then

\section{$\Gamma(\mathrm{Y})$}

stands for the result of substituting label $Y$ for that distinguished occurrence of $X$.

The rule for introducing assumptions has already been given. There are also structural rules. First there is a rule of weakening or thinning. If a conclusion follows from some of our assumptions then we take that as sufficient for it to follow from the whole bunch of them. Inference from a database, for example, is like that: in judging that a statement true given the data we do not require that every single piece of data have been used in its derivation. So we expect and get the rule $\mathbf{K}$ :

$$
\frac{\Gamma(\mathrm{X}): \mathrm{A}}{\Gamma(\Delta(\mathrm{X})): \mathrm{A}}
$$

Then there are further structural rules due to the combinatory properties of set and multiset union. Both operations are associative and commutative, giving rules B:

$$
\frac{\Gamma((\mathrm{X}, \mathrm{Y}), \mathrm{Z}): \mathrm{A}}{\Gamma(\mathrm{X},(\mathrm{Y}, \mathrm{Z})): \mathrm{A}} \quad \frac{\Gamma((\mathrm{X} ; \mathrm{Y}) ; \mathrm{Z}): \mathrm{A}}{\Gamma(\mathrm{X} ;(\mathrm{Y} ; \mathrm{Z})): \mathrm{A}}
$$

and $\mathbf{C}$ :

$$
\frac{\Gamma(\mathrm{X}, \mathrm{Y}): \mathrm{A}}{\Gamma(\mathrm{Y}, \mathrm{X}): \mathrm{A}} \quad \frac{\Gamma(\mathrm{X} ; \mathrm{Y}): \mathrm{A}}{\Gamma(\mathrm{Y} ; \mathrm{X}): \mathrm{A}}
$$

Set union is idempotent, but multiset union is not, so there is a rule $\mathbf{W}$ of contraction for labels combined with commas but not for those combined with semicolons:

$$
\frac{\Gamma(X, X): A}{\Gamma(X): A}
$$

The logical rules giving the meanings of connectives and the like are much as expected, given the attention we are paying to the two modes of combination. Conjunction, as usual, is straightforward:

$$
\begin{gathered}
\frac{X: A}{X, Y: A \wedge B} \wedge I \\
\frac{X: A \wedge B}{X: A} \wedge E \quad \frac{X: A \wedge B}{X: B} \wedge E
\end{gathered}
$$


Note that conjunction, as befits this most extensional of connectives, goes with set combination. Disjunction is marginally more complicated because we are working in a right-handed system. The rules are:

$$
\begin{array}{cr}
\frac{X: A}{X: A \vee B} \vee I & \frac{X: B}{X: A \vee B} \vee I \\
X: A \vee B \quad \Gamma\left(\mathcal{L}_{A}\right): C & \Gamma\left(\mathcal{L}_{B}\right): C \\
\hline & \Gamma(X): C
\end{array}
$$

The reasoning of $\vee \mathrm{E}$ is that if there is some rôle as premise, $\Gamma(\xi)$, which can be played indifferently by $A$ or by $B$ then it can be played by their disjunction or by anything $X$ which entails their disjunction. Thus the logic of conjunction and disjunction is very standard.

The operation of implication forms a kind of logical conditional from its antecedent and its consequent. Such a conditional is a consequence of assumptions if and only if the addition of the antecedent to those assumptions would enable the consequent to be derived. Thus for instance 'All logicians are underpaid' entails 'If McRobbie is a logician then McRobbie is underpaid' because by applying 'All logicians are underpaid' to the additional assumption that McRobbie is a logician we can logically deduce that McRobbie is underpaid. The putting together of major and minor premises here is multiset putting together, for they are interacting, the one being applied to the other, to produce the conclusion 9 Hence the natural rules for the introduction and elimination of implication are:

$$
\begin{gathered}
\frac{X ; \mathcal{L}_{\mathrm{A}}: \mathrm{B}}{\mathrm{X}: \mathrm{A} \rightarrow \mathrm{B}} \rightarrow \mathrm{I} \\
\mathrm{X: \textrm {A } \rightarrow \mathrm { B } : \mathrm { B }} \quad \mathrm{Y}: \mathrm{A}
\end{gathered}
$$

Modus ponens, being the form of $\rightarrow \mathrm{E}$ corresponding straightforwardly to $\rightarrow \mathrm{I}$ on the above motivating remarks, is indeed fundamental to the present account of conditionals as Dummett's challenge requires.

Negation is intuitionistic. The neatest way to incorporate it is to define it in terms of an absurd constant $\perp$ :

$$
\sim A={ }_{\mathrm{df}} A \rightarrow \perp
$$

Then the only negation principle required is the absurdity rule:

$$
\frac{X: \perp}{X: A} \perp \mathbf{E}
$$

${ }^{9}$ For a more extended discussion of this point see $\left[{ }_{13},[14]\right.$. 
The rules for quantifiers are rather like those of classical or intuitionistic free logic, because they need to account for the fact that some of the objects quantified over may exist only at lax degrees of truth. Therefore there is a primitive existence predicate $\mathrm{E}$ ! with the reading that $\mathrm{E} ! \mathrm{t}$ is true to the degree that $t$ denotes something.

As well as E!, the language is taken to have the usual apparatus of variables and function symbols, from which are constructed terms. The story about freedom and bondage is the normal one. In specifying the rules, we use the notation

$$
A_{v}^{t}
$$

to stand for the result of substituting term $t$ for all and only free occurrences of variable $v$ in formula $A$. The rules are:

$$
\begin{aligned}
& \frac{X: A}{X: \forall \vee A} \quad \forall \mathbf{I} \quad \begin{array}{l}
\text { where } v \text { does not } \\
\text { occur free in } X
\end{array} \\
& \frac{X: \forall v A \quad Y: E ! t}{X ; Y: A_{v}^{t}} \forall \mathbf{E} \quad \begin{array}{l}
\text { where } t \text { is free } \\
\text { for } v \text { in } A
\end{array}
\end{aligned}
$$

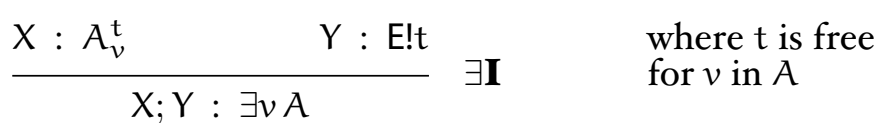

$$
\begin{aligned}
& \frac{X: \exists v A \quad \Gamma\left(\mathcal{L}_{A}\right): B}{\Gamma(\mathrm{X}): \mathrm{B}} \exists \mathbf{E} \quad \begin{array}{l}
\text { where } v \text { does not } \\
\text { occur free in } \Gamma(\mathrm{B})
\end{array}
\end{aligned}
$$

In addition, it is axiomatic that everything exists:

$$
\emptyset: E ! v
$$

Here is a sample proof just to show what proofs look like:

$$
\begin{gathered}
\frac{1: p \rightarrow q \quad 3: p}{1 ; 3: q} \rightarrow \mathbf{E} \quad \frac{2: p \rightarrow r \quad 3: p}{2 ; 3: r} \wedge \mathbf{E} \\
\frac{\frac{(1 ; 3),(2 ; 3): q \wedge r}{(1 ; 2 ; 3),(1 ; 2 ; 3): q \wedge r}}{\frac{1 ; 2 ; 3: q \wedge r}{1 ; 2: p \rightarrow(q \wedge r)} \rightarrow \mathbf{I}(\times \mathbf{2})} \mathbf{W} \\
\frac{1:(p \rightarrow r) \rightarrow(p \rightarrow(q \wedge r))}{1: \mathbf{I}}
\end{gathered}
$$


The structural $\mathbf{K}$ and $\mathbf{W}$ moves in the middle of this proof bear closer inspection. The two applications of $\mathbf{K}$ are necessary in order to unify the two parenthesised sublabels so that $\mathbf{W}$ can apply. Of course, if we were to use this formulation of logic $\mathbf{F}$ in real applications, these low-level appeals to the "algebra of labels" would be elided for conciseness and readability.

As noted, contraction for ';' is not available, as multiset union is not idempotent. Intensional contraction is essential to the classical proofs of all of the following sequents, none of which is provable in $\mathbf{F}$ :

$$
\begin{array}{rll}
p \rightarrow(p \rightarrow q) & \vdash p \rightarrow q \\
p, p \rightarrow q & \vdash q \\
p \rightarrow q, q \rightarrow r & \vdash p \rightarrow r \\
p, \sim q & \vdash & \sim(p \rightarrow q) \\
p \rightarrow \sim p & \vdash & \sim p \\
& \vdash & \sim(p \wedge \sim p) \\
\forall x E ! f x ; \forall x(F x \rightarrow F f x) & \vdash \forall x(F x \rightarrow F f f x)
\end{array}
$$

The last of these is especially pertinent to the sorites argument, for it is the form of the inference from 'One step preserves F' to 'Two steps preserve F'. The second, third and fourth on the above list of $\mathbf{F}$-invalid sequents crucially have commas rather than semicolons on the left. The analogous sequents with semicolons are all easily provable.

\section{Semantics}

To deepen the explanation of system $\mathbf{F}$ we now turn to semantics, the object being to relate the logic to intuitively graspable structures and distinctions. What follows is a modelling in the style of Kripke's semantics for intuitionist logic. Just as intuitionists generally feel that the classical realist "possible worlds" story, even if read in terms of "states of information" or some such, misrepresents them, so $\mathbf{F}$ supporters (when eventually there are such things) will probably hold the present paper to be an example of how not to approach their position. Well, let that be. For the moment our problem is to get some semantic grip on $\mathbf{F}$, in our own heathen tongue if necessary. Such an exercise can at least give us information about the system: for example, it is a way of finding out which are the $\mathbf{F}$-valid inferences.

We first model the propositional fragment of $\mathbf{F}$, since most differences between our logic and the standard brands can be isolated at the propositional level where the model structures are relatively simple. The basis is a set $\mathcal{D}$ of "degrees to which the truth could be stretched". These might well be individuated by reference to statements which they make true (“...just enough to make 
it true that $R(50)$ ", etc.). We then need a relation $\geqslant$ between members of $\mathcal{D}$ and multisets of members of $\mathcal{D}$. Where $M$ is a multiset of degrees and $d$ is a particular degree we write

$$
d \geqslant M
$$

to indicate that $d$ stretches the truth as far as all the members of $M$ put together. The idea of putting together, of course, is inherited from the syntactic concept of applying one assumption or piece of information to another as happens in uses of modus ponens. The degrees are put together in multisets rather than just in sets because for this purpose repetitions count: as we have seen, the results of applying a premise to itself need not be the same as those of taking that premise on its own.

Before going further, it is worthwhile to define a relation of weak compatibility:

$$
\mathrm{d} \odot \mathrm{e}={ }_{\mathrm{df}} \exists x(x \geqslant[\mathrm{~d}, \mathrm{e}])
$$

So $\mathrm{d}$ and $e$ are weakly compatible iff it would not be totally absurd to put them together. The structure then satisfies the postulates $[10$

pI. $\quad d \geqslant[d]$

p2. $d \geqslant M \sqcup N \Rightarrow d \geqslant M$

p3. $\quad e \geqslant N \sqcup[d], d \geqslant M \Rightarrow e \geqslant N \sqcup M$

p4. $d \geqslant M \sqcup N \Rightarrow \exists e_{(e \geqslant M)} \exists f_{(f \geqslant N)} d \geqslant[e, f]$

Postulates pr and p2 should be fairly obvious given the intended reading. Postulate $\mathrm{p} 3$ makes the ordering relation appropriately transitive and monotonic. Postulate $\mathrm{p}_{4}$ is harder to see intuitively. It says in effect that every partition of a multiset (into parts $M$ and $N$ ) is "witnessed" by a pair of degrees ( $e$ and $f$ ). The claim that every multiset $M$ stretches the truth to a particular degree $\delta_{M}$

$$
\forall \mathrm{d}\left(\mathrm{d} \geqslant\left[\delta_{M}\right] \Leftrightarrow \mathrm{d} \geqslant \mathrm{M}\right)
$$

would allow $\mathrm{p} 4$ to be simplified to

$\mathrm{p}^{+} . \quad \delta_{\mathrm{M}} \sqcup \mathrm{N} \geqslant\left[\delta_{\mathrm{M}}, \delta_{\mathrm{N}}\right]$

This, however, would be properly stronger than p4; the weaker version is sufficient for the completeness proof below. In what follows the structure

$$
\langle\mathcal{D}, \geqslant\rangle
$$

shall be known as an $\mathbf{F}$ frame.

\footnotetext{
${ }^{10}$ Notation for multisets is like that for sets, but square. So a multiset may be given in extension by listing betwen square brackets, and multiset intersection and union are written with square cap and cup respectively. When being careful, we may distinguish between the null set $\emptyset$ and the null multiset $\pitchfork$.
} 
It is not part of the definition of an $\mathbf{F}$ frame that there should exist a zero degree of stretch, or absolute truth. A frame is said to be with zero iff it contains a degree 0 such that

$$
\forall \mathrm{d} d \geqslant[0]
$$

From the formal point of view, absolute truth is an optional extra, since the presence or absence of a zero does not affect the stock of theorems or validated inferences.

An interpretation is a function I assigning to each atomic formula a subset of $\mathcal{D}$ such that for any atom $A$ and any $e \geqslant[d]$

$$
d \in I(A) \Rightarrow e \in I(A)
$$

We write

$$
\mathrm{d} \models_{\mathrm{I}} A
$$

to mean that degree $d$ of stretching would suffice to make formula $A$ true for interpretation I. In what follows the subscript will be omitted for clarity.

The recursive clauses giving the meanings of connectives are:

$$
\begin{aligned}
& \text { I. } \quad \mathrm{d} \models \mathrm{p}_{\mathrm{k}} \quad \Longleftrightarrow \mathrm{d} \in \mathrm{I}\left(\mathrm{p}_{\mathrm{k}}\right) \\
& \text { 2. } \quad \mathrm{d} \models A \wedge \mathrm{B} \Longleftrightarrow \mathrm{d} \models A \text { and } \mathrm{d} \models \mathrm{B} \\
& \text { 3. } \mathrm{d} \models A \vee \mathrm{B} \Longleftrightarrow \mathrm{d} \models A \text { or } \mathrm{d} \models \mathrm{B} \\
& \text { 4. } \quad d \models A \rightarrow B \Longleftrightarrow \forall e_{(e \models=A)} \forall f_{(f \geqslant[d, e])} f \models B \\
& \text { 5. } \quad \mathrm{d} \models \sim A \Longleftrightarrow \forall e_{(\mathrm{d} \odot e)} e \not \forall A
\end{aligned}
$$

This definition of the relation $\models$ extends in a natural way from formulae to bunches of formulae. A degree realises a set of bunches iff it realises every member of that set, and where $B$ is a multiset of bunches $d \models B$ iff $d \geqslant M$ for some multiset $M$ of degrees where there is a one-one mapping $\mu$ of $B$ onto $M$ such that for all $X$ in $B, \mu(X) \models X$.

Clauses 4 and 5 need comment. Clause 4 reflects the reading given to the conditional when motivating the natural deduction system. What it is for $d$ to yield 'If $A$ then $B$ ' just is that by putting it together with any $e$ which yields $A$ we can get B. How much more clearly could modus ponens be made constitutive of the meaning of 'if'? Clause 5 results from clause 4 on the definition of negation as implying absurdity. To be in a position to deny $A$ is not just to fail to be in a position to assert $A$, for we want the possibility of becoming more generous as to what counts as assertible without thereby contradicting ourselves.

Formula $A$ is said to be true for I iff for every degree $d, d \models_{I} A$. It is valid iff true for every interpretation. Sequent $\langle X, A\rangle$ is valid iff on every interpretation, for every degree $d$, if $d \models X$ then $d \models A$. Soundness and completeness theorems for the propositional logic $\mathbf{F}$ are fairly straightforward $\amalg$ In a frame with

\footnotetext{
${ }^{\text {II }}$ See the next section for these and other proofs. See [3] or [2] for the general technique of such completeness proofs. The notion of an $\mathbf{F}$ frame given in the present paper is adapted from
} 
zero, of course, truth for an interpretation is just a matter of holding at degree 0 . Extension of the story to deal with quantifiers is less simple, but interesting, as will be explained below after some exploration of the propositional logic.

The account of the sorites inference is much as expected. Assertibility in the ordinary sense is a vague notion: there will be a fuzzy set of "small degrees of stretch" rather close to absolute truth with the property that anything true to such a degree is ordinarily assertible: criticism of one who asserted it would be misplaced, for instance. All of the premises of the sorites hold at some points $\mathrm{d}$ corresponding to degrees like that, but the conclusion does not because holding-at-a-point is not closed under detachment. The real modus ponens is built into the modelling condition for $\rightarrow$ (condition 4 above), meeting Dummett's challenge head-on.

The above semantic account is designed to keep what is valuable in the Łukasiewicz approach while rejecting the dross. The idea of letting the degrees of departure from truth accumulate in step with the application of assumptions is retained, as is the distinction between the real and the pseudo modus ponens. Total order and specifically arithmetical properties are however abandoned. Moreover the logic $\mathbf{F}$, unlike the Łukasiewicz system, is deductiontheoretically natural. It also has characteristics which make possible a novel approach to the problems of higher order vagueness, but to approach these we need some metatheorems.

\section{Completeness And Other theorems}

The main result of this section is that exactly the same sequents are valid in $\mathbf{F}$ frames as are provable in $\mathbf{F}$ as formulated above, justifying the nomenclature.

Firstly we add to the language an intensional conjunction connective $\circ$ subject to the introduction and elimination rules

$$
\begin{gathered}
\frac{X: A \quad Y: B}{X ; Y: A \circ B} \circ \mathbf{I} \\
\frac{X: A \circ B \quad \Gamma\left(\mathcal{L}_{A} ; \mathcal{L}_{B}\right): C}{\Gamma(X): C} \circ \mathbf{E}
\end{gathered}
$$

Secondly we give a more formal definition of a "bunch" of formulae. Bunches are of two kinds: S-bunches and M-bunches as follows.

the work of Routley, Meyer and others on Kripke models for the relevant logics. The fact that their three-place relation can be used to give semantics for fuzzy logics of the Łukasiewicz type has often been noted, first perhaps by Lakoff in [6]. It may be worth pointing out that $\mathbf{F}$ is not in any sense a relevant logic, as $\mathrm{P} \vdash_{\mathrm{F}} \mathrm{Q} \rightarrow \mathrm{P}$.

${ }^{12}$ This connective is known in linear logic as "tensor product" and in the relevant logic literature as "fusion", as it "fuses" its conjuncts into a new object which does not easily decompose back into them. It has algebraically natural properties such as associativity and commutativity, making it easier to handle semantically than the implication connective. 
I. A formula is both an S-bunch and an M-bunch.

2. Any set of M-bunches is an S-bunch.

3. Any multiset of S-bunches is an M-bunch.

4. Nothing is a bunch except by finitely many applications of the above clauses.

Let $\beta$ be a bunch. If $\beta$ is an M-bunch, define $\beta^{s}$ as $\{\beta\}$; otherwise let $\beta^{s}$ be $\beta$. Conversely, if $\beta$ is any S-bunch, let $\beta^{m}$ be defined as $[\beta]$; otherwise let $\beta^{m}$ be $\beta$.

Next we define what it is for a finite sequent to be provable. A sequent is an ordered pair consisting of a bunch of formulae (its antecedents) and a single formula (its succedent). By a labelling scheme we mean an assignment to each formula $A$ of a label $\mathcal{L}_{A}$. A derivation under a labelling scheme is one in which each axiom (assumption) is a formula labelled according to the scheme. Then each molecular label $\sigma$ corresponds to a bunch of formulae $\mathcal{B}(\sigma)$ :

I. Where $A$ is a formula, $B\left(\mathcal{L}_{A}\right)=A$

2. $\mathcal{B}(X, Y)=\mathcal{B}(X)^{s} \cup \mathcal{B}(Y)^{s}$

3. $\mathcal{B}(X ; Y)=\mathcal{B}(X)^{m} \sqcup \mathcal{B}(Y)^{m}$

Although the function $\mathcal{B}$ is not one-one, it may be inverted in a sense. For any finite bunch $X$ we may choose as $\mathcal{B}^{\prime}(X)$ the first label $\mathcal{L}$ in some sensible order (e.g. lexicographic order) such that $\mathcal{B}(\mathcal{L})=X$. A finite sequent $\langle\beta, A\rangle$ is provable iff under every labelling scheme there is a derivation of the labelled formula $\mathcal{B}^{\prime}(\beta): A$.

To define what it is for an infinite sequent to be provable, we introduce the notion of a finitisation of a given bunch and of a sequent.

I. Any formula is a finitisation of itself.

2. Where $X_{1} \ldots X_{n}$ are finitisations of $Y_{1} \ldots Y_{n}$ and $\left\{Y_{1} \ldots Y_{n}\right\} \cup Z$ is an $S$ bunch, $\left\{X_{1} \ldots X_{n}\right\}$ is a finitisation of $\left\{Y_{1} \ldots Y_{n}\right\} \cup Z$.

3. Where $X_{1} \ldots X_{n}$ are finitisations of $Y_{1} \ldots Y_{n}$ and $\left[Y_{1} \ldots Y_{n}\right] \sqcup Z$ is an $M$ bunch, $\left[X_{1} \ldots X_{n}\right]$ is a finitisation of $\left[Y_{1} \ldots Y_{n}\right] \sqcup Z$.

4. Sequent $\langle\beta, A\rangle$ is a finitisation of sequent $\left\langle\beta^{\prime}, A\right\rangle$ iff $\beta$ is a finitisation of $\beta^{\prime}$.

5. Nothing is a finitisation except as a consequence of this definition.

In other words, the finitisations of a sequent are those finite sequents which can be converted into it by (possibly repeated and possibly infinitary) applications of weakening. Then we say that a sequent is provable just in case some finitisation of it is provable. 
We next define for each finite bunch $X$ of formulae the characteristic formula $X^{\star}$ as follows

I. Where $A$ is a formula, $A^{\star}$ is $A$.

2. $\left\{X_{1} \ldots X_{n}\right\}^{\star}$ is $X_{1}^{\star} \wedge \ldots \wedge X_{n}^{\star}$.

3. $\left[X_{1} \ldots X_{n}\right]^{\star}$ is $X_{1}^{\star} \circ \ldots \circ X_{n}^{\star}$.

Evidently the finite sequent $\langle X, A\rangle$ is provable iff $\left\langle X^{\star}, A\right\rangle$ is provable, which is the case iff $\mathcal{B}^{\prime}(X): A$ is obtainable by a derivation whose assumptions $\mathcal{L}_{B}: B$ all use unstructured (atomic) labels, and iff $X^{\star} \rightarrow A$ is a theorem of $\mathbf{F}$ (provable with the null label). Evidently also, if $Y$ is a finitisation of $X$ then

$$
X \vdash Y^{\star}
$$

Next we define the canonical truth set $\mathcal{T}$. No atomic formula is in $\mathcal{T}$, and:

$$
\begin{aligned}
A \wedge B \in \mathcal{T} & \Longleftrightarrow A \in \mathcal{T} \text { and } B \in \mathcal{T} \\
A \circ B \in \mathcal{T} & \Longleftrightarrow A \in \mathcal{T} \text { and } B \in \mathcal{T} \\
A \vee B \in \mathcal{T} & \Longleftrightarrow A \in \mathcal{T} \text { or } B \in \mathcal{T} \\
\sim A \in \mathcal{T} & \Longleftrightarrow \quad \sim A \text { and } A \notin \mathcal{T} \\
A \rightarrow B \in \mathcal{T} & \Longleftrightarrow A \vdash B \text { and if } A \in \mathcal{T} \text { then } B \in \mathcal{T}
\end{aligned}
$$

The turnstile represents provability in $\mathbf{F}$.

LEMma 4 If $\mathrm{A} \rightarrow \mathrm{B} \in \mathcal{T}$ then $\Gamma(\mathrm{A})^{\star} \rightarrow \Gamma(\mathrm{B})^{\star} \in \mathcal{T}$.

For proof, suppose $A \rightarrow B \in \mathcal{T}$. Clearly if $\Gamma(A)^{\star} \in \mathcal{T}$ then each formula in $\Gamma(A)$ is in $\mathcal{T}$, so each formula in $\Gamma(B)$ is in $\mathcal{T}$, so $\Gamma(B)^{\star} \in \mathcal{T}$. The proof is therefore completed by a quick induction on the complexity of $\Gamma$ showing that if $A \rightarrow B$ is a theorem of $\mathbf{F}$ then so is $\Gamma(A)^{\star} \rightarrow \Gamma(B)^{\star}$. This is left to the reader.

LEMma 5 If $\Gamma(A \vee B)^{\star} \in \mathcal{T}$ then either $\Gamma(A)^{\star} \in \mathcal{T}$ or $\Gamma(B)^{\star} \in \mathcal{T}$.

Proof: Another induction on the complexity of $\Gamma$. In the base case $\Gamma(A)$ is $A$ and there is nothing to prove. One of the induction cases is $\Gamma(A)=\Delta(A), X$. If $\Gamma(A \vee B)^{\star} \in \mathcal{T}$ then $\Delta(A \vee B)^{\star} \in \mathcal{T}$ and $X^{\star} \in \mathcal{T}$. But $\Delta$ is less complex than $\Gamma$, so either $\Delta(A)^{\star} \in \mathcal{T}$ or $\Delta(B)^{\star} \in \mathcal{T}$, so either $\Delta(A)^{\star} \wedge X^{\star} \in \mathcal{T}$ or $\Delta(B)^{\star} \wedge X^{\star} \in \mathcal{T}$ as required. The other induction cases are similar.

THEOREM 6 (Metacompleteness) $\vdash A \Longleftrightarrow A \in \mathcal{T}$.

Equivalently, for finite $X, X \vdash A \Longleftrightarrow X^{\star} \rightarrow A \in \mathcal{T}$

${ }^{13}$ We suppose that the multi-ary conjunctions are to be read sensibly: say, with the conjuncts in lexicographic order and associated to the left. 
Proof: Proof is by induction in both directions. Right to left is an induction on the complexity of $A$ and is trivial. Left to right is an induction on the length of proofs in $\mathbf{F}$. The base case is a derivation of length $I$, where $X^{\star} \rightarrow A$ is just $A \rightarrow A$ which is clearly in $\mathcal{T}$. The induction step is very case-ridden as each rule has to be verified. The case of $\vee E$ is hardest and will serve as an illustration. Suppose

$$
\begin{aligned}
& X^{\star} \rightarrow A \vee B \\
& \Gamma(A)^{\star} \rightarrow C \\
& \Gamma(B)^{\star} \rightarrow C
\end{aligned}
$$

are all in $\mathcal{T}$. Clearly if either $\Gamma(A)^{\star}$ or $\Gamma(B)^{\star}$ is in $\mathcal{T}$ then $C$ is in $\mathcal{T}$, so by lemma 5 if $\Gamma(A \vee B)^{\star}$ is in $\mathcal{T}$ then so is $C$. Clearly $\Gamma(A \vee B)^{\star} \vdash C$. Therefore $\Gamma(A \vee B)^{\star} \rightarrow C \in \mathcal{T}$. By lemma 4 therefore $\Gamma(X)^{\star} \rightarrow C \in \mathcal{T}$. The rest of the cases are similar but easier and are left as another exercise.

COROLLARY 7 For any formulae A and B, if $\vdash \mathrm{A} \vee \mathrm{B}$ then either $\vdash \mathrm{A}$ or $\vdash \mathrm{B}$.

Proof: by inspection of theorem 6 and the definition of $\mathcal{T}$.

By an $\mathbf{F}$-theory we understand a set of formulae closed under $\mathbf{F}$ entailment. Theory $\theta$ is prime iff whenever it contains a disjunction it contains at least one of the disjuncts, detached iff closed under the pseudo modus ponens inference

$$
\{\mathrm{A} \rightarrow \mathrm{B}, \mathrm{A}\} \Longrightarrow \mathrm{B}
$$

and normal if both prime and detached. Clearly the set $\mathcal{F}$ of theorems of $\mathbf{F}$ itself is a normal $\mathbf{F}$-theory. We expect that the world (everything that is the case) is another. We do not expect all theories to be normal: most scientific theories for example are not prime, and the set of sentences assertible to some given high degree, though a theory, is not detached. Still, prime theories are the ones that give the extensional connectives their full truth functional due, and detached ones do the same for intensional connectives, so as logicians we take such theories to be of the most formal interest.

Where $\Sigma$ is a bunch of formulae and $\theta$ is any set of formulae we define the $\theta$ closure of $\Sigma$ by

$$
\mathcal{C}_{\theta}(\Sigma)=\{A: \Sigma ; \theta \vdash A\}
$$

Clearly an equivalent condition would be

$$
\mathcal{C}_{\theta}(\Sigma)=\left\{\mathrm{B}: \exists \mathrm{A}_{(\Sigma \vdash \mathrm{A})}(\theta \vdash \mathrm{A} \rightarrow \mathrm{B})\right\}
$$

Note also that $\mathcal{C}_{\mathcal{F}}(\Sigma)$ is just $\mathcal{C}_{\emptyset}(\Sigma)$, the set of logical consequences of $\Sigma$. For any set $\sigma$ of formulae and multiset $\Sigma$ of sets of formulae we define ' $\sigma$ is as strong as $\Sigma$ given $\theta^{\prime}$ by

$$
\sigma \geqslant_{\theta} \Sigma={ }_{\mathrm{df}} \mathcal{C}_{\theta}(\Sigma) \subseteq \sigma
$$

A set $\sigma$ is said to be a $\theta$-theory iff $\sigma=\mathcal{C}_{\theta}([\sigma])$. 
THEOREM 8 Let $\theta$ be any normal $\mathbf{F}$-theory. Let $\Pi$ be the set of prime $\theta$-theories. Then $\left\langle\Pi, \geqslant_{\theta}\right\rangle$ is an $\mathbf{F}$ frame with zero $\theta$.

Proof: Proof is a matter of checking the postulates. The cases of pr and $\mathrm{p} 2$ are trivial. Postulate $\mathrm{p}_{3}$ needs a bit of work. Let $\Sigma$ and $\Theta$ be multisets of prime $\theta$-theories. By the definition of logical consequence, the multiset union $\Sigma \sqcup \Theta$ entails $A$ iff for some finitisation $\phi$ of $\Theta, \Sigma \sqcup\left[\phi^{\star}\right]$ entails $A$. But $\phi^{\star} \in \mathcal{C}_{\mathcal{F}}(\Theta)$, so by weakening, $\Sigma \sqcup\left[\mathcal{C}_{\mathcal{F}}(\Theta)\right]$ entails $A$. That is to say

$$
\mathcal{C}_{\mathcal{F}}(\Sigma \sqcup \Theta) \subseteq \mathcal{C}_{\mathcal{F}}\left(\Sigma \cup\left[\mathcal{C}_{\mathcal{F}}(\Theta)\right]\right)
$$

By the unpacking of definitions this fact extends from $\mathcal{C}_{\mathcal{F}}$ to $\mathcal{C}_{\theta}$. Consequently if $\mathcal{C}_{\theta}(\Theta) \subseteq \sigma$ and $\mathcal{C}_{\theta}(\Sigma \sqcup[\sigma]) \subseteq \pi$ then $\mathcal{C}_{\theta}(\Sigma \sqcup \Theta) \subseteq \pi$ which is to say p3. For p4 we use Zorn's lemma. Suppose

$$
\pi \geqslant{ }_{\theta} \Sigma \sqcup \Theta
$$

where $\pi$ is a prime $\theta$-theory and $\Sigma$ and $\Theta$ are multisets of such. Then clearly

$$
\pi \geqslant{ }_{\theta}\left[\complement_{\theta}(\Sigma), \mathcal{C}_{\theta}(\Theta)\right]
$$

Chains of theories are bounded above by their unions, and if the union of a chain of theories features as a sub-bunch on the left of a provable sequent then it can be replaced there by some member of the chain because, consequence being compact, the sequent can be finitised. Let $\alpha$ be a maximal super- $\theta$-theory of $\mathcal{C}_{\theta}(\Sigma)$ such that

$$
\pi \geqslant{ }_{\theta}\left[\alpha, \mathcal{C}_{\theta}(\Theta)\right]
$$

as guaranteed by Zorn's lemma. Similarly, let $\beta$ be a maximal super- $\theta$-theory of $\mathcal{C}_{\theta}(\Theta)$ such that

$$
\pi \geqslant \theta[\alpha, \beta]
$$

It is quite easy to check that $\alpha$ and $\beta$ are prime, for suppose neither $A$ nor $B$ is in $\alpha$. By its maximality,

$$
\begin{aligned}
& (\alpha, A) ; \mathcal{C}_{\theta}(\Theta) ; \theta \vdash C \\
& (\alpha, B) ; \mathcal{C}_{\theta}(\Theta) ; \theta \vdash D
\end{aligned}
$$

for some $C$ and $D$ neither of which is in $\pi$. By the primeness of $\pi, C \vee D$ is not in $\pi$ either. But by $\vee \mathrm{I}$ and $\vee \mathrm{E}$

$$
(\alpha, A \vee B) ; \mathcal{C}_{\theta}(\Theta) ; \theta \vdash C \vee D
$$

whence $A \vee B$ is not in $\alpha$. The argument that $\beta$ is prime is similar.

LEMMA 9 (Squeeze) Let $\theta$ be a set offormulae. For any $\theta$-theory $\alpha$ and for any formulae $\mathrm{A}$ and $\mathrm{B}$, if $\mathrm{A} \rightarrow \mathrm{B} \notin \alpha$ then there exist prime $\theta$-theories $\beta$ and $\gamma$ with $\mathrm{A} \in \beta$ and $\mathrm{B} \notin \gamma$ such that $\mathrm{C}_{\theta}([\alpha, \beta]) \subseteq \gamma$. 
Proof: let $\alpha$ be a $\theta$-theory not containing $A \rightarrow B$. Define $\beta_{0}$ as $\mathcal{C}_{\theta}(\{A\})$. Note that $\mathrm{B} \notin \mathcal{C}_{\theta}\left(\left[\alpha, \beta_{0}\right]\right)$ since otherwise $\theta ; \alpha ; A \vdash B$, whence $A \rightarrow B \in \alpha$ contrary to the supposition. Use Zorn's lemma to choose a maximal $\theta$-theory $\beta$ such that $\beta_{0} \subseteq \beta$ and $\mathrm{B} \notin \mathcal{C}_{\theta}([\alpha, \beta])$. Then $\beta$ is prime, for let $C$ and $\mathrm{D}$ be formulae not in $\beta$. Then

$$
\begin{aligned}
& \theta ; \alpha ;(\beta, C) \vdash \mathrm{B} \\
& \theta ; \alpha ;(\beta, D) \vdash \mathrm{B}
\end{aligned}
$$

Hence by $\vee \mathrm{E}$

$$
\theta ; \alpha ;(\beta, C \vee D) \vdash B
$$

whence $C \vee D \notin \beta$. Use Zorn's lemma again to deliver a maximal supertheory $\gamma$ of $\mathcal{C}_{\alpha}(\beta)$ not containing B. By a similar argument, $\gamma$ is again prime, so $\beta$ and $\gamma$ suffice for the lemma.

THEOREM IO Let $\theta$ be a normal $\mathbf{F}$-theory. Let the canonical $\theta$ frame be defined as in theorem 8 For each atomic formula $\mathrm{p}_{\mathrm{k}}$ let $\mathrm{I}\left(\mathrm{p}_{\mathrm{k}}\right)$ be the set of prime $\theta$-theories containing $p_{k}$. Then for every formula $A$ and for every degree $\mathrm{d}$ in the frame, $\mathrm{d} \models_{1} \mathrm{~A}$ iff $\mathrm{A} \in \mathrm{d}$.

Proof: Proof by induction on the construction of $A$. The only case presenting any problem is $A=B \rightarrow C$, which is cleared up by the squeeze lemma.

Theorem IO suffices to show the completeness of $\mathbf{F}$ for validity in $\mathbf{F}$ frames, for as noted the set $\mathcal{F}$ is a normal theory whence the canonical model to which it gives rise falsifies all nontheorems together and provides for every invalid sequent $X: A$ a degree $d$ such that $d \models X$ but $d \not \models A$. The converse soundness theorem is proved by (tedious) induction over the length of proofs which is left to the reader.

\section{Reflections}

The canonical frame

$$
\langle\Pi, \geqslant \emptyset\rangle
$$

and the canonical interpretation in it for which for all $\theta$ and $A$

$$
\theta \models A \Longleftrightarrow A \in \theta
$$

in some sense constitute the intended model of the logic $\mathbf{F}$. Let is call them the Lindenbaum frame and the Lindenbaum model since they correspond to the Lindenbaum algebra obtained as the quotient algebra under the congruence relation of provable equivalence on the algebra of formulae. In the Lindenbaum model there is no higher-order vagueness: every proposition has its value exactly, for in effect every proposition takes itself, or its logical equivalence class, as value. So higher order imprecision stops somewhere, the Lindenbaum model giving an upper bound on where it stops. There is no vagueness in the matter of 
whether one thing logically entails another, however vague those things themselves may be. Hence we can model the logic of imprecise discourse precisely without falling foul of the meta-sorites arguments and without doing the least violence to the facts.

It is a very special feature of logics like our system $\mathbf{F}$ that the Lindenbaum frame is a frame in the fullest sense. The same picture cannot be drawn with respect to the Łukasiewicz continuum-valued logic, for instance, for in such cases there is no analogue of theorem 6 and corollary 7 . The set of theorems of Łukasiewicz's logic is not a normal theory for it fails to be prime. For example,

$$
(\mathrm{P} \rightarrow \mathrm{Q}) \vee(\mathrm{Q} \rightarrow \mathrm{P})
$$

is a theorem though of course neither disjunct is. Thus by re-working fuzzy logic as $\mathbf{F}$ not only have we secured a formulation deserving the title of natural deduction (and even better proof-theoretic properties, for which see below) but we have the makings of a solution to one of the hardest problems in the field, that of marrying lower-order vagueness with higher-order precision.

There is, however, a price to be paid for such a neat escape from such a traditionally stubborn difficulty. In the Lindenbaum model the truths-those propositions which hold at the zero world - are just the logical truths. And in reality we hardly expect the logical truths to be the only truths. It is good that there should be models verifying no contingent truths, so that pure logic is not required to claim that there are truths beyond logic; but of course there should also be models looking like reality, in which there are many truths of other sorts. We therefore expect that the world will consist of more than logic, though we do not expect logic itself to tell us what more there is to the truth. A couple of formal facts are of some help in making the semantics of $\mathbf{F}$ into a more adequate account.

FACT II Let $S$ be any set of atomic formulae. Then there is a model in which the true atomic formulae are exactly those in $S$.

Proof: Fact $\mathrm{II}$ is trivial, as it is for classical logic and for any other sensible system: in the Lindenbaum frame, let $I(p)$ be $\{0\}$ if $p \in S$ and $\emptyset$ otherwise.

This is a rather meagre step albeit in the right direction, as the Lindenbaum frame still draws more distinctions than we really want. In that frame the "degrees" are just all the prime theories, whereas we should like the degree structure too to be largely a function of what is the case rather than something dictated simply by logic itself. For another small step in the right direction, then:

FACT I2 Suppose $A$ is not deducible from set $S$ of atomic formulae by means of logical entailment and detachment. Then there is a model in which for any atomic $\mathrm{P}, 0 \models \mathrm{P} \Longleftrightarrow \mathrm{P} \in \mathrm{S}$ but $\mathrm{O} \not \models A$ and in which for all degrees $\mathrm{d}$ and $e$ if $e \notin d$ then there is some $B$ such that $d \models B$ but $B \notin \mathcal{C}_{S}(\{C: e \models C\})$. 
Proof: For proof, let $\theta$ be the closure of $\mathcal{C}_{\mathcal{F}}(S)$ under detachment. Clearly $\theta$ is a detached theory whose atomic theorems are exactly the members of $S$. Then re-work the proof of theorem 6 above, using $\mathcal{T}_{\theta}$ in place of $\mathcal{T} . \mathcal{T}_{\theta}$ is defined like $\mathcal{T}$ except that atoms are in it iff they are in $\theta$ and the deducibility parts of the negation and implication clauses are re-written as $\theta \vdash \sim A$ and $\theta ; A \vdash B$ respectively. The upshot is that $\mathcal{T}_{\theta}$ is identical with $\theta$, and so is a normal theory agreeing with $\mathrm{S}$ on atoms. Theorem $\mathrm{IO}$ then delivers the result.

In the light of this we are assured of a model in which the true atomic sentences are just those free of any vagueness which would be accounted true by a classical (or perhaps an intuitionistic) truth-theorist. Moreover, there are no "degrees" in its frame except for those needed to make distinctions between formulae not equivalent given those atoms. We can do even better, using Zorn's lemma to beef up an arbitrary theory to a maximal (hence prime) supertheory still avoiding a given nontheorem and then cutting back in the manner of fact I2 to give an intuitively "large" normal theory on which can be based a frame. Firm technical results on the availability of truth-like frames are somewhat elusive, however.

It is worth pausing here to note the possibility of strengthening $\mathbf{F}$ by adding the "double negation elimination":

$$
\frac{X: \sim \sim A}{X: A} \text { DNE }
$$

This gives a system I shall dub $\mathbf{F}^{*}$. It is shown in [IO] that theorem 6 goes through for $\mathbf{F}^{*}$ though in a slightly more complicated form because of the non-constructive account of negation. Semantics for $\mathbf{F}^{*}$ may be obtained easily from those for $\mathbf{F}$ by adding Sylvan's star operation to frames as in [3], re-writing the evaluation clause for negation

$$
\mathrm{d} \models \sim A \quad \Longleftrightarrow \quad \mathrm{d}^{*} \not A \mathrm{~A}
$$

as usual.

The logic $\mathbf{F}^{* *}$, otherwise known as the "logic of constructible falsity" [8], is a proper supersystem of $\mathbf{F}^{*}$ with delightfully simple semantics. A frame for $\mathbf{F}^{* *}$ is just a partially ordered set of "degrees". At each degree, each formula may be true, false or neither. All values (true or false) are inherited by stronger degrees. We may let $\mathcal{V}(A, d)$ be the value (if any) of $A$ at $d$. The interpretation conditions for connectives are as follows.

$$
\begin{aligned}
& \mathcal{V}(A \wedge B, d)=T \Longleftrightarrow \mathcal{T}(A, d)=T \text { and } \mathcal{V}(B, d)=T \\
& \mathcal{V}(A \wedge B, d)=F \quad \Longleftrightarrow \mathcal{F}(A, d)=F \text { or } \mathcal{V}(B, d)=F \\
& \mathcal{V}(A \vee B, d)=T \Longleftrightarrow \mathcal{T}(A, d)=T \text { or } \mathcal{V}(B, d)=T \\
& \mathcal{V}(A \vee B, d)=F \quad \Longleftrightarrow \mathcal{V}(A, d)=F \text { and } \mathcal{V}(B, d)=F \\
& \mathcal{V}(\sim A, d)=T \quad \Longleftrightarrow \mathcal{T}(A, d)=F
\end{aligned}
$$




$$
\begin{aligned}
\mathcal{V}(\sim A, d)=\mathrm{F} \Longleftrightarrow & \mathcal{V}(A, d)=T \\
\mathcal{V}(A \rightarrow B, d)=T \Longleftrightarrow & \text { for all e such that } e \geqslant d, \\
& \text { if } \mathcal{V}(A, e)=T \text { then } \mathcal{V}(B, e)=T \text { and } \\
& \text { if } \mathcal{V}(B, e)=F \text { then } \mathcal{V}(A, e)=F \\
\mathcal{V}(A \rightarrow B, d)=F \Longleftrightarrow & \mathcal{V}(A, d)=T \text { and } \mathcal{V}(B, d)=F
\end{aligned}
$$

It was once conjectured that $\mathbf{F}^{* *}=\mathbf{F}^{*}$ but this turned out to have been a vain hope ${ }^{14}$ The formula

$$
\mathrm{P} \circ \mathrm{Q} \rightarrow . \mathrm{P} \circ \mathrm{P} \circ \mathrm{Q} \vee \mathrm{P} \circ \mathrm{Q} \circ \mathrm{Q}
$$

is valid in $\mathbf{F}^{* *}$ but is not a theorem of $\mathbf{F}^{*}$. In fact, its addition to $\mathbf{F}^{*}$ suffices to axiomatise $\mathbf{F}^{* *}$. The investigation of $\mathbf{F}^{*}$ and $\mathbf{F}^{* *}$ lies beyond the scope of the present paper; for now it suffices to say that they represent logical points of view close to that associated with $\mathbf{F}$ and could be made the focus of a less anti-realist account of reasoning with vagueness.

\section{First ORDER LOGIC}

We now turn from the propositional fragment of $\mathbf{F}$ to the full logic including quantification theory. The introduction and elimination rules for quantifiers were given earlier along with those for the connectives, but so far we have not considered semantics for the predicate logic. In fact, the necessary amendments to the propositional semantics are very much what might be expected given the intuitionistic cast of $\mathbf{F}$.

A frame is what it was before, with the addition of a function $\mathcal{E}$ assigning to each $\mathrm{d}$ in $\mathcal{D}$ a nonempty set of objects $\varepsilon_{\mathrm{d}}$ forming its domain of quantification. Where $e \geqslant[\mathrm{~d}]$, we allow that there may be objects in $\mathcal{E}_{e}$ which are not in $\mathcal{E}_{\mathrm{d}}$, since the criteria for existence may be relaxed along with those for truth as we move from $\mathrm{d}$ to $e$. That is, there is an extra heredity postulate for domains:

p5. $\quad e \geqslant[d] \Rightarrow \mathcal{E}_{\mathrm{d}} \subseteq \mathcal{E}_{e}$

An interpretation is now a more complicated function assigning values at degrees to function and predicate symbols again subject to the heredity condition. A function symbol is assigned at each degree a partial function on the domain of that degree, and a predicate symbol is assigned a relation defined on the domain. That is:

$$
\begin{aligned}
& \mathrm{I}_{\mathrm{d}}\left(\mathrm{f}_{\mathrm{k}}^{\mathrm{n}}\right): \Sigma \longrightarrow \mathcal{E}_{\mathrm{d}} \text { for some } \Sigma \subseteq\left(\mathcal{E}_{\mathrm{d}}\right)^{\mathrm{n}} \\
& \mathrm{I}_{\mathrm{d}}\left(\mathrm{F}_{\mathrm{k}}^{\mathfrak{n}}\right) \subseteq\left(\mathcal{E}_{\mathrm{d}}\right)^{\mathrm{n}}
\end{aligned}
$$

Moreover, interpretation is hereditary with respect to the partial order of strength between degrees:

\footnotetext{
${ }^{14}$ For this observation I am indebted to Tim Surendonk.
} 


$$
\begin{aligned}
& e \geqslant[\mathrm{~d}] \Rightarrow \mathrm{I}_{\mathrm{d}}\left(\mathrm{f}_{k}^{\mathfrak{n}}\right) \subseteq \mathrm{I}_{e}\left(\mathrm{f}_{k}^{\mathfrak{n}}\right) \\
& e \geqslant[\mathrm{~d}] \Rightarrow \mathrm{I}_{\mathrm{d}}\left(\mathrm{F}_{k}^{\mathfrak{n}}\right) \subseteq \mathrm{I}_{e}\left(\mathrm{~F}_{k}^{\mathfrak{n}}\right)
\end{aligned}
$$

On the basis of this we may define denotation $\delta_{d}^{I}$ for terms:

$$
\begin{aligned}
& \delta_{d}^{I}(v) \in \mathcal{E}(d) \\
& \delta_{d}^{I}\left(f_{k}^{n} t_{1} \ldots t_{n}\right)=I_{d}\left(f_{k}^{n}\right)\left(\delta_{d}^{I}\left(t_{1}\right) \ldots \delta_{d}^{I}\left(t_{n}\right)\right)
\end{aligned}
$$

So a term either fails to denote for a given degree or it denotes an individual from the domain of that degree, and continues to denote the same individual for all stronger degrees. The new base clause for the inductive definition of realisation reads:

I.

$$
\mathrm{d} \models \mathrm{F}_{\mathrm{k}}^{\mathrm{n}} \mathrm{t}_{1} \ldots \mathrm{t}_{\mathrm{n}} \Longleftrightarrow\left\langle\delta_{\mathrm{d}}^{\mathrm{I}}\left(\mathrm{t}_{1}\right) \ldots \delta_{\mathrm{d}}^{\mathrm{I}}\left(\mathrm{t}_{\mathrm{n}}\right)\right\rangle \in \mathrm{I}_{\mathrm{d}}\left(\mathrm{F}_{\mathrm{k}}^{\mathrm{n}}\right)
$$

and it is necessary to add two new clauses to deal with the quantifiers. For these, as usual let a $v$-variant of interpretation I be any interpretation differing from I at most in the values it assigns (at degrees) to variable $v$. Then

6. $\quad \mathrm{d} \models_{\mathrm{I}} \forall v \mathrm{~A} \Longleftrightarrow$ for every $v$-variant $\mathrm{I}^{\prime}$ of I, for every e such that $e \geqslant[\mathrm{~d}], e \models_{\mathrm{I}^{\prime}} \mathrm{A}$

7 .

$$
\mathrm{d} \models_{\mathrm{I}} \exists v A \Longleftrightarrow \text { for some } v \text {-variant } \mathrm{I}^{\prime} \text { of } \mathrm{I}, \mathrm{d} \models_{\mathrm{I}^{\prime}} A
$$

Sequent $X: A$ is valid iff for every interpretation $I$ in every frame, for every degree $d$ :

$$
\mathrm{d} \models_{\mathrm{I}} \mathrm{X} \Rightarrow \mathrm{d} \models_{\mathrm{I}} \mathrm{A}
$$

Our next aim is to prove first order $\mathbf{F}$ sound and complete for quantificational $\mathbf{F}$ frames as just defined. The key to the soundness proof is a heredity lemma.

LEMMA I3 (Heredity) In any interpretation $\mathrm{I}$, for any degrees $\mathrm{d}$ and e and for any formula $\mathrm{A}$, if $\mathrm{e} \geqslant[\mathrm{d}]$ and $\mathrm{d} \models_{\mathrm{I}}$ A then $\mathrm{e} \models_{\mathrm{I}} \mathrm{A}$.

Proof: Proof is by induction on the complexity of $A$ and is perfectly straightforward. In the base case $A$ is atomic. Since values of everything subatomic are by stipulation inherited, this case is trivial. The induction cases $A=\sim B$ and $A=\forall v B$ are also trivial, and the conjunction and disjunction cases pose no real problem. Where $A=\exists v B$, if $d \models_{I} A$ then there is some a-variant $\mathrm{I}^{\prime}$ of I such that $\mathrm{d} \models_{\mathrm{I}^{\prime}} \mathrm{B}_{v}^{\mathrm{a}}$. On induction hypothesis therefore $e \models_{\mathrm{I}^{\prime}} \mathrm{B}_{v}^{\mathrm{a}}$ whence $e \models_{I} A$. The remaining case is that in which $A$ is $B \rightarrow C$. Suppose so, and that $\mathrm{d} \models_{\mathrm{I}} A$. Then suppose $f \models_{\mathrm{I}} \mathrm{B}$ and $\mathrm{g} \geqslant[e, f]$. By p3, $g \geqslant[\mathrm{~d}, \mathrm{f}]$, so $g \models_{\mathrm{I}} \mathrm{C}$. That is, $e \models_{I} A$.

THEOREM I4 (Soundness) Every first order sequent provable in $\mathbf{F}$ is valid in quantificational $\mathbf{F}$ frames.

Proof: Proof is by induction on the length of proofs in $\mathbf{F}$ and is omitted here as none of the many cases is particularly interesting. 
The completeness theorem is proved much as in the propositional case, with the usual complications to take care of quantifiers. The metacompleteness theorem continues to hold, for closed formulae, with the extra clauses on the canonical truth set $\mathcal{T}$ :

$$
\begin{aligned}
& \forall v A \in \mathcal{T} \quad \Longleftrightarrow \quad \forall v A \text { and for every closed term } t, A_{v}^{\mathrm{t}} \in \mathcal{T} \\
& \exists v A \in \mathcal{T} \quad \Longleftrightarrow \quad A_{v}^{\mathrm{t}} \in \mathcal{T} \text { for some closed term } \mathrm{t}
\end{aligned}
$$

As before, this enables the Lindenbaum model of the logic to be used to refute all nontheorems together. The theories which correspond to degrees are of course those which are not only prime but also saturated in that whenever they contain an existential formula they also contain a ground instance of it. Hence in constructing such theories for the priming and squeeze lemmas, care must be taken to add instances of any existential formulae included. Verification of the postulates is fairly straightforward, except for the witness postulate p4. The problem here is that we need to be assured that in "beefing up" the witnesses into prime and saturated theories $e$ and $f$, the instances of existentials which get added can all be chosen so as not to destroy the condition that if $[e, f] \vdash A$ then $A \in d$. The fact that so assures us is the pair of confinement laws

$$
\begin{aligned}
& \mathrm{A} \wedge \exists v \mathrm{~B} \vdash \exists v(\mathrm{~A} \wedge \mathrm{B}) \\
& \mathrm{A} \circ \exists v \mathrm{~B} \vdash \exists v(\mathrm{~A} \circ \mathrm{B})
\end{aligned}
$$

where $v$ does not occur free in $A$. Then for instance when we have multisets $X$ and $Y$ of theories and a saturated theory $d$ such that $\mathcal{C}_{\mathcal{F}}(X \sqcup Y) \subseteq \mathrm{d}$ and we wish to extend $\mathcal{C}_{\mathcal{F}}(Y)$ to a prime and saturated theory $f$ such that $\mathcal{C}_{\mathcal{F}}(X \sqcup[\mathrm{f}]) \subseteq \mathrm{d}$, any existential in $f$ may be instantiated by some term which instantiates it in d. Without going into further detail, therefore, we announce

THEOREM I5 (Completeness) Every first order sequent which is valid in quantificational $\mathbf{F}$ frames is provable in $\mathbf{F}$.

Having a fully articulated formal modelling of the logic of vague expressions makes it possible to clarify certain philosophical questions. For example, there has been much discussion in the recent literature of the question of whether, and in what sense, there might be vague objects as opposed to vague predicates. It seems that several non-equivalent cases need to be distinguished. One way in which an object might be vague is in the possession of blurred or imprecise boundaries. This, after all, was Frege's original paradigm case of vagueness: an area which shades off gradually into its surroundings like a photographic image out of focus. Clearly there are lots of vague objects in this sense. Clouds and crowds come to mind immediately. Or think of the Sahara desert, or the Thames estuary. In such cases it is a vague matter which smaller items are parts of the object. At a sufficiently microscopic level, even physical objects of the table-and-chair variety are like this. In terms of the opening 
examples, we find vague objects of this sort if we are prepared to count my childhood (i.e. that stretch of time during which I was a child) or the red part of the Dummett strip as an object.

A more radical notion is that of an object whose existence is vague, which exists only to a degree. Observe that the semantic picture sketched for system $\mathbf{F}$ allows for objects vague in this sense too, as domains may increase in size as we become more lax as to what counts as true. What sort of object could be vague in this sense? Presumably one whose existence depends on its being of a certain kind. Thousands of years ago there was fertile grassland where the Sahara now is; at that time the Sahara did not exist. The difficulty of picking a time at which it came into existence is not merely epistemological but is due to the fact that it exists only insofar as it is a desert, and the predicate '.. is a desert' is a vague one. An object could be vague in this sense without having vague boundaries. A crowd might be bounded by the walls of a room, for example, and yet its existence ("qua crowd", we like to say) supervenes on the spatial arrangement of persons, so it depends on whether so few people so disposed in such a space counts as a crowd.

Most of the recent discussion of vague objects has focussed on the question of whether identity statements, presumably using proper names for such objects, can be vague ${ }^{15}$ The logic of identity has not been incorporated into $\mathbf{F}$ as formulated above, but clearly it can be, and there is some choice as to the postulates. Several types of "vague identity statements" might then be distinguished. Firstly, where $n$ denotes an object whose existence is vague as in the second sense above, the sentence

$$
n=n
$$

will be realised only by degrees for which that object exists and so will be vague. Secondly, there may be terms which fail to denote at some points and come to denote at some others. They may do this even if the objects they denote are already in the domains of the former points. So for example an object may exist at all degrees, and perhaps be denoted by name $n_{1}$ at all degrees, but may come to be denoted by $n_{2}$ only at relatively lax degrees. In such a case the identity statement

$$
\mathrm{n}_{1}=\mathrm{n}_{2}
$$

will be vague. Thirdly, it is coceivable that objects which exist and are distinct at some degree can become identical at some more generous degree. The semantics of $\mathbf{F}$ given above do not allow for such conflation, but it is an easy matter to incorporate it without changing the stock of valid sequents. Although semantics for identity statements have not yet been detailed, this possibility of conflation would seem to ensure that they behave semantically much like any other atomic formulae. Clearly there are other options at the semantic level,

${ }^{15}$ See for example the seminal discussion by Evans [4] 
the most extreme of which would be to insist on constant domains (the same for all degrees) in order to make identity rigid. Such proposals, naturally, would affect what could be modeled in the way of vague identity and vague entity.

At any rate, non-constant domains (and therefore degrees of existence) are needed to falsify unprovable sequents such as

$$
\forall x(F x \vee G x): \forall x F x \vee \exists x G x
$$

The usual intuitionist story as to why these are not wanted makes essential reference to construction. It is because the construction process need not be completed at any point and because only constructed objects count as existing that variable domains occur and provide counter-examples to inferences like the above. At a given point only Fs and no Gs may have been constructed, while at later points some non-Fs could be constructed provided they are all Gs. Some variant of this account emerges from the simple view that in mathematics nothing except provability counts as truth or from the recent more sweeping verificationist thought that quite generally nothing except knowability counts as truth. There is nothing specifically epistemic about the given motivation for $\mathbf{F}$. The analogue of investigation-dependent construction is stretch-dependent truth. Clearly, though, an anti-realist account of reference for terms and for sentences is congenial enough to the $\mathbf{F}$ perspective. On such an account it is we who "stretch the truth" by setting our criteria with different degrees of laxness. The naturalness of such a thought sits well with the fact that the logic $\mathbf{F}$ is a subset of the intuitionist theory. Pursuing the philosophical ramifications of the realist-antirealist debate with respect to vaguness is another project to be postponed, however.

\section{Proof theory AND DECIDABILITY}

At the risk of boring any reader diligent enough to have got this far, there now follows yet another presentation of the logic $\mathbf{F}$. This time it is as a cut-free system to be styled GF in honour of Gentzen ${ }^{16}$ Proofs in this style still relate sequents, but now instead of elimination rules for connectives and quantifiers on the right we have more introduction rules for their occurrences on the left, so that proofs proceed only by construction, never by destruction.

We consider only finite sequents, allowing that they may be empty or single on the right and have any finite bunch on the left. The structural rules are as expected:

$$
\frac{\Gamma(\mathrm{X}, \mathrm{X}):[\mathrm{A}]}{\Gamma(\mathrm{X}):[\mathrm{A}]} \mathbf{W} \vdash
$$

\footnotetext{
${ }^{\text {I6 }}$ The system here called $\mathbf{G F}$ is roughly that called $\mathbf{L} \mathbf{L}_{\mathrm{DBCK}}$ in [II]. The cut elimination theorem is adapted from the one given in that paper.
} 


$$
\begin{array}{cc}
\frac{\Gamma(\mathrm{X}, \mathrm{Y}):[\mathrm{A}]}{\Gamma(\mathrm{Y}, \mathrm{X}):[\mathrm{A}]} \mathbf{C} \vdash & \frac{\Gamma(\mathrm{X} ; \mathrm{Y}):[\mathrm{A}]}{\Gamma(\mathrm{Y} ; \mathrm{X}):[\mathrm{A}]} \mathbf{C} \vdash \\
\frac{\Gamma(\mathrm{X}):[\mathrm{A}]}{\Gamma(\Delta(\mathrm{X})):[\mathrm{A}]} \mathrm{K} \vdash & \frac{\mathrm{X}:}{\mathrm{X}: \mathrm{A}} \vdash \mathbf{K} \\
\frac{\Gamma(\mathrm{X},(\mathrm{Y}, \mathrm{Z})):[\mathrm{A}]}{\Gamma((\mathrm{X}, \mathrm{Y}), \mathrm{Z}):[\mathrm{A}]} \mathrm{B} \vdash & \frac{\Gamma(\mathrm{X} ;(\mathrm{Y} ; \mathrm{Z})):[\mathrm{A}]}{\Gamma((\mathrm{X} ; \mathrm{Y}) ; \mathrm{Z}):[\mathrm{A}]} \mathbf{B} \vdash
\end{array}
$$

Here ' $[A]$ ' is either a formula or nothing. Note that while the structural rules of reassociation, weakening and exchange hold for both types of premise combination, contraction (W) holds only for extensional combination.

The logical rules should again occasion no surprise:

$$
\begin{aligned}
& \frac{\Gamma(A, B): C}{\Gamma(A \wedge B): C} \& \vdash \quad \frac{X: A \quad Y: B}{X, Y: A \wedge B} \vdash \& \\
& \frac{\Gamma(A ; B): C}{\Gamma(A \circ B): C} \circ \vdash \quad \frac{X: A \quad Y: B}{X ; Y: A \circ B} \vdash \circ \\
& \frac{\Gamma(A): C \quad \Gamma(B): C}{\Gamma(A \vee B): C} \vee \vdash \quad \frac{X: A}{X: A \vee B} \vdash \vee \quad \frac{X: B}{X: A \vee B} \vdash \vee \\
& \frac{X: A \quad \Gamma(B): C}{\Gamma(A \rightarrow B ; X): C} \rightarrow \vdash \quad \frac{X ; A: B}{X: A \rightarrow B} \vdash \rightarrow
\end{aligned}
$$

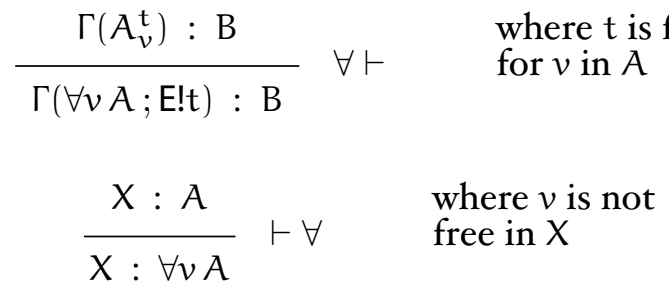

$$
\begin{aligned}
& \frac{\Gamma(A): B}{\Gamma(\exists v A): B} \quad \exists \vdash \quad \begin{array}{l}
\text { where } v \text { is not } \\
\text { free in } \Gamma(B)
\end{array}
\end{aligned}
$$




$$
\begin{array}{cl}
X: A_{v}^{t} & \text { where } t \text { is free } \\
X ; E ! t: \exists v A & \text { for } v \text { in } A
\end{array}
$$

The axioms of $\mathbf{G F}$ are all sequents

$$
A \vdash A
$$

where $A$ is an atomic formula, together with the special sequents

$\vdash E ! v$

and

$$
\perp \vdash
$$

A proof in $\mathbf{G F}$ is a finite tree of sequents each of which is either an axiom or is related to its children by one of the above rules. It is a proof of the sequent at its root.

Proof that every sequent derivable in $\mathbf{G F}$ is derivable in $\mathbf{F}$ is easy. For the empty right-hand side we have to substitute something appropriate: $\perp$ is appropriate. Some of the cases require inductions on the complexity of bunch contexts $\Gamma$ but these are quite straightforward, especially given the (easily established) admissibility in $\mathbf{F}$ of the rule $\mathbf{C u t}$

$$
\frac{X: A \quad \Gamma(A): B}{\Gamma(X): B}
$$

The converse proof, that everything provable in $\mathbf{F}$ is provable in $\mathbf{G F}$ is harder. Again it goes through on a standard induction over lengths of proofs, and again the key to making this work is proving the admissibility of Cut. This time, however, the admissibility of Cut is less obvious. To prove it we actually establish the apparently stronger result that the rule $\mathbf{M i x}$ is admissible. A mix, for $\mathbf{G F}$, is an inference of the form

$$
\begin{array}{ll}
X: A & \Gamma(A \ldots A):[B] \\
\hline & \Gamma(X \ldots X):[B]
\end{array}
$$

where

$$
\Gamma(\alpha \ldots \beta)
$$

is the generalisation of our previous notation indicating a bunch in which $\alpha$ occurs, possibly many times in different contexts, and $\beta$, occurs in one distinguished context. The mix may replace any number of occurrences of the "mix formula" A by occurrences of X. Ordinary Cut is the special case in which only one occurrence is replaced. 
As usual, elimination is proved by double induction on rank (greatest number of steps since the introduction of any of the occurrences of the mix formula) and degree (length of the mix formula). It will not be detailed here. However, it is worth noting the form taken by the degree-reduction case where there are several occurrences of the mix formula. By way of illustration, consider the case of a mix whose mix formula $A \rightarrow B$ is introduced on both left and right immediately before the mix:

$$
\frac{\frac{X ; A: B}{X: A \rightarrow B} \vdash \rightarrow \quad \frac{\Gamma(A \rightarrow B \ldots B): C \quad Y: A}{\Gamma(A \rightarrow B \ldots(A \rightarrow B ; Y)): C} \rightarrow \vdash}{\Gamma(X \ldots(X ; Y)): C} \text { mix }
$$

Here the left premise comes by the left introduction rule for the mix formula, but the mix may also replace other occurrences. After the transformation, there remains a mix on $A \rightarrow B$ and two new mixes have been added:

$$
\frac{\frac{X ; A: B}{X: A \rightarrow B} \vdash \rightarrow \Gamma(A \rightarrow B \ldots B): C}{\frac{\Gamma(X \ldots B): C}{\frac{\Gamma(X \ldots(X ; A)): C}{\Gamma(X \ldots(X ; Y)): C}}{ }^{\text {mix }} Y: A} \text { mix }
$$

The mix on $A \rightarrow B$ has lower rank than the original and on induction hypothesis is therefore eliminable, while the other two may have any rank but have lower degree and so again are eliminable. Similar things happen in the other degree reduction cases, and the rank reduction cases pose no special difficulties. After a certain amount of the usual checking of cases, therefore, we may announce: THEOREM I6 (Mix Elimination) Let GFM be the system resulting from $\mathbf{G F}$ by the addition of the rule Mix. Then every sequent provable in $\mathbf{G F M}$ is provable in $\mathbf{G F}$.

Proof: Proof is by induction on degree and rank in that order of priority, and is left as an exercise for the reader, who may use the above illustration as a template.

COROLla RY I7 A sequent is provable in $\mathbf{G F}$ iff it is provable in $\mathbf{F}$.

Proof: it is not difficult to turn a proof in GF into a proof of the same sequent in $\mathbf{F}$, remembering that formulae in the left side of sequents are to be represented by their atomic labels, and so must be assumed in the labelled deductive system in order that the labels be assigned. Then each rule of $\mathbf{G F}$ can be emulated quite straightforwardly in $\mathbf{F}$. In the converse manner, every proof in $\mathbf{F}$ 
can be imitated in GFM (using cuts). The cut (or mix) elimination theorem closes the circle by showing that a proof in GFM can be turned into one in GF.

The fact that $\mathbf{F}$ has a cut-free equivalent in $\mathbf{G F}$ is useful in many ways. For example, it affords an alternative proof of corollary 7 , that $\mathcal{F}$ is a prime theory, for the shortest cut-free proof of a sequent

$$
\vdash A \vee B
$$

must use the rule $\vdash \vee$ last, whence one of the disjuncts must be a theorem. For the most important application of $\mathbf{G F}$, however, we need some more definitions.

First, let us refer to the written representations of bunches on the left of sequents as structures. A structure of the form $X, Y$ is extensional while a formula (standing in for its singleton) or a structure of the form $X ; Y$ is intensional. The constituents of a structure are such of its intensional substructures as are not themselves proper substructures of any of its intensional substructures. So the sole constituent of an intensional structure is itself while the constituents of $X, Y$ are those of $X$ together with those of $Y$. We think of the constituents as a sequence rather than a set, because the structure is a sequentially written object. A structure is reduced iff no substructure has two occurrences of the same item among its constituents. It is semi-reduced iff no constituent has more than two occurrences of the same item among its constituents. A sequent is reduced or semi-reduced iff its left side is, and a proof is reduced or semireduced iff every sequent in it is. By applying structural rules we can easily find for any sequent $S$ a reduced sequent $r(S)$ which is provable iff $S$ is provable.

LEMMA I8 Every provable reduced sequent has a semi-reduced proof.

Proof: Proof is by induction on the construction of proofs. The key fact making this induction trivial is that for any instance of a rule

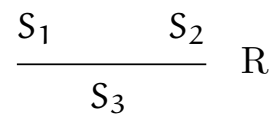

(where $S_{2}$ may be null) either $r\left(S_{1}\right)=r\left(S_{3}\right)$ or there is a derivation

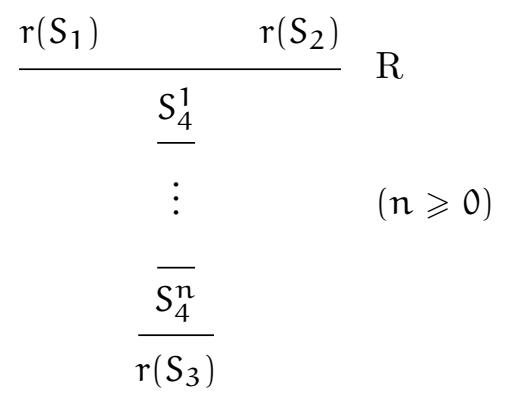


in which each $S_{4}^{i}$ is semi-reduced and in which all the subsidiary inferences are by structural rules. This is established simply but laboriously by cases. The lemma is immediate.

A sequent in the present syntactically-oriented sense is supposed to represent a sequent in the former set-theoretic sense. It will help at this point to define the equivalence between the two notions. For each structure $X$ we define the corresponding bunch $\mathcal{B}(\mathrm{X})$ as follows.

I. $\mathcal{B}(A)$ is just $A$.

2. Where $X$ and $Y$ are extensional structures $\mathcal{B}(X, Y)$ is $\mathcal{B}(X) \cup \mathcal{B}(Y)$ and $\mathcal{B}(\mathrm{X} ; \mathrm{Y})$ is $[\mathcal{B}(\mathrm{X}), \mathcal{B}(\mathrm{Y})]$

3. Where $X$ and $Y$ are intensional structures $\mathcal{B}(X ; Y)$ is $\mathcal{B}(X) \sqcup \mathcal{B}(Y)$ and $\mathcal{B}(X, Y)$ is $\{\mathcal{B}(X), \mathcal{B}(Y)\}$

4. Where $X$ is an extensional structure and $Y$ is an intensional structure $\mathcal{B}(X, Y)$ is $\mathcal{B}(X) \cup\{\mathcal{B}(Y)\}$, as is $\mathcal{B}(Y, X)$, while $\mathcal{B}(X ; Y)$ and $\mathcal{B}(Y ; X)$ are $[\mathcal{B}(X)] \sqcup \mathcal{B}(Y)$.

We shall say that two sequents are abstractly equivalent iff they have the same conclusion and their premises correspond to the same bunch. For instance, $A ; B: C$ is abstractly equivalent to $(A ;(B, B)),(B ; A): C$ in virtue of the idempotence of set union and the commutativity of multiset union. Next we define the length of a sequent $X: A$ to be the number of occurrences of subformulae in $\mathcal{B}(X)$ and $A$.

theorem in Propositional $\mathbf{G F}$ is decidable. Hence propositional $\mathbf{F}$ is decidable.

Proof: Proof is by induction on the length of sequents. It is obvious that short sequents (of length 2 or less) are decidable. To decide a given sequent of greater length we first note that it is provable iff its reduced equivalent is provable, and that (by lemma I8) iff it has a semi-reduced proof. So we may restrict attention to semi-reduced sequents. Now only finitely many semi-reduced sequents are abstractly equivalent to the given one, and clearly these can be effectively generated. The structural rules B, C and W do not take us out of an abstract equivalence class, so if our sequent has a proof then one of its semi-reduced abstract equivalents has a proof whose last move is not B, C or W. But all the other rules strictly increase length and so reversing them takes us to shorter sequents whose provability we can decide. Moreover, there are at a given point only finitely many ways for rules to apply (in jargon, the proof search tree has the finite fork property), so after finitely much checking of possibilities we are done. 
Time was when contributors to philosophical logic needed to do no more than demonstrate the appropriateness of a logic for the abstract description of validity across a chosen range. In those days it sufficed that language suitably regimented had indeed the structure of the presented system. Now, though, correctness is not enough. In the terms of the new electronic pragmatism, the truth may not be pure but it had better be simple. It is therefore necessary at this point to pass a few remarks on the suitability of $\mathbf{F}$ for automated reasoning purposes.

For reasons which have always escaped me $\sqrt{17}$ it is widely held that a good first step towards practicability is that the propositional logic be decidable. Propositional $\mathbf{F}$ is decidable. The decision procedure given in the last section is in the form of a proof search method which delivers a proof if there is one and otherwise delivers the information that there is not. In the quantified case, too, a straightforward proof search based on the Gentzen formulation will find a proof if one exists. Many of the methods of [I5] can be used to improve on the grossly inefficient behaviour of the naïve implementation of GF. In particular, the structural rule of contraction can be omitted, its effects being written into slightly generalised forms of the other rules. It is also easy to adapt the matrix testing technique of $\left[{ }_{5}\right]$ ] to prune the proof search tree radically. Work remains to be done on the details of automating deductions in $\mathbf{F}$ and $\mathbf{F}^{*}$, but at least we know what kind of work that is, and there is no shortage of recent research on automated theorem proving from which it can start. It appears that as regards automation, $\mathbf{F}$ is in at least as good shape as most nonstandard logics, and indeed since contraction is the root of a good deal of computational evil in this area it may well be in better shape than some.

\section{Conclusion}

The indicated strategy for us "deviant" logicians, then, is to set out a decent alternative view such as that associated with system F, meeting Dummett's challenge in full, and in the light of such a coherent alternative to demand the reasons for thinking modus ponens in its vicious form to be valid. On Dummett's own admission that inference leads from assertible premises to absurd conclusions, so what can be meant by the claim that it is valid, and what sorts of evidence of its validity are forthcoming? This paper has presented a system of logic meeting all sensible criteria of adequacy. $\mathbf{F}$ has a very natural and graspable formulation giving the quantifiers and connectives the deductive properties they should have; it has a set of plausible semantic interpretations; it has a straightforward cut-free equivalent; at the propositional level it is decidable; it lends itself to automated reasoning. On the general grounds that consistent

\footnotetext{
${ }^{17}$ Church long ago demonstrated that all worthwhile logics are undecidable, so decidability is not a necessary condition for anything that really matters. Nor of course is it a sufficient condition, for it is consistent with decidability that the worst case (and indeed average case) complexity of all decision algorithms be such as to render them unworkable.
} 
solutions to philosophical difficulties are to be welcomed where available, it seems at least that the burden of more argument is shifted back to the orthodox view 18

\section{REFERENCES}

[I] M. DUmmetт. Wang's paradox. Synthese, 30:30I-324, I975.

[2] J. M. DUNN. Relevance logic and entailment. In D. GABBAY and F. GÜNTHNER, editors, Handbook of Philosophical Logic I I I. Reidel, Dordrecht, I986.

[3] F. R. ROUtLey et al. Relevant Logics and their Rivals. Ridgeview, Atascadero CA, 1982.

[4] G. evans. Can there be vague objects? Analysis, 38:208, 1978.

[5] K. FINE. Vagueness, truth and logic. Synthese, 30:265-300, 1975.

[6] G. LAKOFF. Hedges, a study in meaning criteria and the logic of fuzzy concepts. Fournal of Philosophical Logic, 2:458-508, 1973.

[7] R. K. Meyer. A general gentzen system for implicational calculi. Relevance Logic Newsletter, I:I89-201, 1976.

[8] D. NELSON. Constructible falsity. Fournal of Symbolic Logic, I4:I4-26, 1949.

[9] w. v. O. Quine. Word and Object. mit Press, Cambridge ma, I960.

[io] J. K. Slaney. Reduced models for relevant logics without wi. Notre Dame Fournal of Formal Logic, 28:395-407, 1987.

[II] J. K. Slaney. Solution to a problem of Ono and Komori. Fournal of Philosophical Logic, I8:IO3-III, I989.

[I2] J. K. SLANEy. Vagueness revisited. Technical Report TR-ARP-I5-88, Automated Reasoning Project, Australian National University, 1989.

[13] J. K. Slaney. A general logic. Australasian fournal of Philosophy, 68:74-88, I990.

[I4] J. K. Slaney and R. K. MEYer. Logic for two: The semantics of distributed substructural logics. In Proceedings of the First International foint Conference on 2ualitative and 2uantitative Practical Reasoning (ECSQARUFAPR'97), pages 554-567, 1997 .

${ }^{18}$ This paper had its origins in a series of seminar presentations in $1983-4$. The main part of the material was subsequently presented to the Scots Philosophical Club in Edinburgh in 1987 , and re-worked in 1988 to be released as a technical report [I2] which appeared in 1989 . Although that report has been circulating quite widely for over ten years, it has never been published. I am grateful to Greg Restall for urging me to prepare it for publication now. 
[i5] p. в. thistlewaite, m. A. mCrobiie, and r. k. meyer. Automated Theorem-Proving in Non-Classical Logics. Pitman, London, 1988.

[i6] T. Williamson. Vagueness. Routledge, London, i994.

[I7] C. J. G. WRIGHT. On the coherence of vague predicates. Synthese, 30:324365,1975 .

[I8] C. J. G. WRIGHT. Language mastery and the sorites paradox. In G. EVANS and J. MCDOwell, editors, Truth and Meaning: Essays in Semantics, pages 223-247. Clarendon, Oxford, 1976. 
The Australasian fournal of Logic (ISSN I448-5052) disseminates articles that significantly advance the study of logic, in its mathematical, philosophical or computational guises. The scope of the journal includes all areas of logic, both pure and applied to topics in philosophy, mathematics, computation, linguistics and the other sciences.

Articles appearing in the journal have been carefully and critically refereed under the responsibility of members of the Editorial Board. Only papers judged to be both significant and excellent are accepted for publication.

The journal is freely available at the journal website at

$$
\text { http://www.philosophy.unimelb.edu.au/ajl/ }
$$

All issues of the journal are archived electronically at the journal website.

SuBSCRIPTIONS Individuals may subscribe to the journal by sending an email, including a full name, an institutional affiliation and an email address to the managing editor at ajl-editors@unimelb.edu.au Subscribers will receive email abstracts of accepted papers to an address of their choice. For institutional subscription, please email the managing editor at ajl-editors@unimelb.edu.au.

Complete published papers may be downloaded at the journal's website at http: //www.philosophy.unimelb.edu.au/ajl/ The journal currently publishes in pdf format.

Submission The journal accepts submissions of papers electronically. To submit an article for publication, send the $\mathrm{LT}_{\mathrm{E}} \mathrm{X}$ source of a submission to a member of the editorial board. For a current list of the editorial board, consult the website.

The copyright of each article remains with the author or authors of that article. 